\title{
EDITORIAL
}

\section{EQUIDAD EN SALUD: UN RETO PARA EUROPA}

\author{
Ildefonso Hernández Aguado \\ Dirección General de Salud Pública y Sanidad Exterior. Ministerio de Sanidad y Política Social. Madrid
}

Hemos comprobado que ideas clave para la mejora de la salud de las poblaciones, como las de Thomas McKeown o las de Geoffrey Rose, se difunden muy lentamente ${ }^{1 .}$ Así mismo, la puesta en práctica en los servicios de salud de ideas conformadas en el campo de la salud pública es tarda y difícil. Por ejemplo, y aunque en su día fuesen momentáneamente atractivas para el conjunto del sistema tanto las ideas sobre la organización de unos servicios sanitarios orientados hacia la promoción y la prevención, como las de unos servicios sanitarios guiados por los resultados en salud ${ }^{2}$, los intentos de aplicarlas se quedaron en intenciones o pasaron casi desapercibidos por los grupos dirigentes de los servicios de salud, más interesados en resolver los problemas más inmediatos. En España esto ha sido así a pesar de que algunos de estos principios se plasmasen en la norma fundamental de nuestro sistema de salud ${ }^{3}$. No obstante, las distintas aproximaciones realizadas han tenido éxitos parciales, y hoy la posición y el prestigio de la salud pública permiten afrontar nuevos retos.

Uno de ellos es ampliar su alcance para influir y actuar más allá de los servicios sanitarios, y conseguir que la equidad y la salud se sitúen en el corazón de otras políticas además de la sanitaria. El conocimiento actual muestra que nuestra salud viene perfilada por los determinantes sociales en su sentido más amplio, y que por ello, las ganancias en salud se consiguen modificando en el sentido apropiado las políticas educativas, sociales, ambientales, económicas, laborales, etc. El sentido apropiado incluye la reducción constante de las desigualdades y del gradiente social en salud. La equidad efectiva es consustancial a la salud pública y no es posible mejorar la salud de la población en su conjunto si no hay equidad. No es sólo un requisito ético, lo que es primordial, sino además una necesidad práctica, ya que las sociedades cuyas políticas han tendido a la equidad obtienen un mayor rendimiento en todos los sentidos.

Así pues, se afronta un reto de mayor alcance que imbuir de los principios de la salud pública a los servicios sanitarios y que desarrollar las acciones propias de los servicios de salud pública. Se trata de ir más allá del campo sanitario para persuadir a otros ámbitos políticos a que orienten sus acciones hacia ganancias en salud y equidad. Todavía existen muchas barreras, aún no hemos ideado mecanismos estimulantes para que el trabajo intersectorial sea la opción preferible, ni procedimientos imaginativos para deshacer los cercados burocráticos y afrontar los problemas de formas coordinadas.

Pero hay soluciones. La salud pública tiene el prestigio suficiente para influir y con- 
seguir que la salud suba peldaños en la agenda política. Además, cuenta con un desarrollo científico adecuado para que esta influencia parta de su mejor tradición: basar sus mejores acciones en una adecuada información. Ahí reside la intención de la propuesta española para la Presidencia Española de la Unión Europea (UE) en el primer semestre de 2010, en mostrar la necesidad de monitorizar no sólo los desenlaces de salud y las conductas individuales relacionadas, sino también los determinantes sociales de la salud como diana adecuada de las políticas públicas.

Pretendemos que los dirigentes sanitarios europeos trabajen para situar la salud y la equidad en la agenda del resto de las políticas europeas y de los Estados Miembros, mostrando que esta aproximación puede ser eficiente y sostenible, y que se pueden desarrollar sinergias y obtener co-beneficios intersectoriales.

Afortunadamente esta propuesta parte de antecedentes sólidos. El informe final de la Comisión Organización Mundial de la Salud para los Determinantes de la Salud «Subsanar las desigualdades en una generación» ${ }^{4}$ dirigido por Michael Marmot y publicado en Mayo del 2008, supuso un avance notable en la legitimación del estudio de las Desigualdades en Salud, y de las relaciones entre la política y la salud. Así mismo, la adopción de la resolución WHA 62.14 en la $62^{\mathrm{a}}$ asamblea Mundial de la Salud supuso un claro llamamiento a los Estados Miembros para «Reducir las inequidades sanitarias actuando sobre los determinantes sociales de la salud» ${ }^{5}$.

En los últimos años, también en la UE se ha constituido un Grupo de Expertos en Determinantes Sociales y Desigualdades en Salud. Asimismo, las iniciativas particulares de varias Presidencias de la UE Bélgica 2001, Reino Unido 2005, Finlandia 2006 y Portugal 2007 han contribuido en los últimos años a la priorización y desarrollo de la equidad en salud en la agenda europea.
Recientemente, en Octubre de 2009, la Comisión Europea ha adoptado la Comunicación: «Solidaridad en materia de salud: reducción de las desigualdades en salud en la UE», lo que supone un gran avance hacia el compromiso de los Estados Miembros con la equidad en salud 6 .

La aportación española a la Presidencia Europea es sólo una pieza más de la acción necesariamente obstinada de la salud pública; nada está ganado y cada actuación sólo debe ser el preámbulo de las siguientes. Por ello, debemos seguir trabajando en todos los ámbitos de influencia potencial de la salud pública y abogar perseverantemente por la equidad y la salud de la población. En el seno de los propios servicios sanitarios hay espacios para ganar equidad efectiva consiguiendo mayor intensidad de servicios a quien más lo necesita.

\section{BIBLIOGRAFÍA}

1. Alvarez-Dardet C, Ruiz MT. Thomas McKeown and Archibald Cochrane: a journey through the diffusion of their ideas. BMJ. 1993 May 8; 3066887: 1252-5.

2. OMS 2000. Informe sobre la Salud en el Mundo 2000. Mejorar el desempeño de los Sistemas de Salud; http://www.who.int/whr/2000/en/whr00 _es.pdf

3. Ley 14/1986, de 25 de abril, General de Sanidad. BOE del 29 de abril de 1986.

4. CSDH. Closing the gap in a generation: health equity through action on the social determinants of health. Final Report of the Commission on Social Determinants of Health. Geneva: World Health Organization; 2008.

5. World Health Assembly World Health Assembly resolution WHA62.14 on Reducing health inequalities through action on social determinants of health. Geneva: World Health Organization; 2009.

6. European Commission. Communication from the commission to the European Parliament, the Council, the European Economic and Social Committee and the Committee of the Regions on «Solidarity in health: Reducing health inequalities in the EU». Brussels: European Commission; 2009. 